\title{
SURFACE SUBSIDENCE MONITORING AND ANALYZING IN BEIJING BASED ON INTERFEROMETRIC POINT TARGET ANALYSIS
}

\author{
Yi Sun ${ }^{\mathrm{a}, \mathrm{b}}$ Lijun $\mathrm{Lu}^{\mathrm{b}}$ \\ a Shandong University of Science and Technology, Qingdao, China 260910949@qq.com \\ ${ }^{\mathrm{b}}$ Key Laboratory of Geo-Informatics of State Bureau of Surveying and Mapping, Chinese Academy of Surveying and Mapping, \\ Beijing,China
}

Commission III, WG III/6

KEY WORDS: interferometric point target analysis(IPTA); surface subsidence; permanent scatterers(PS); baseline refinement; atmospheric delay; temporal decorrelation; geometrical decorrelation;

\begin{abstract}
:
Temporal and geometrical decorrelation often prevents SAR interferometry from being an operational tool for surface deformation monitoring. Moreover, atmospheric disturbances can strongly compromise the accuracy of the results. In order to solve the above problem. In this paper, Interferometric Point Target Analysis (IPTA) technology was used to obtain surface deformation information with an area of $700 \mathrm{~km}^{2}$ in Beijing. In the experiment, 20 TerraSAR-X images from October 2014 to June 2016 were selected to monitor the surface subsidence in Beijing and higher resolution and higher accuracy WorldDEM obtained by DLR was used to simulated terrain phase, so as to register the high-resolution TerraSAR-X image. The final results show the surface subsidence tendency of Beijing urban from October 2014 to June 2016: in the center of monitor area surface tends to be stable within approximately subsidence rate of $3 \mathrm{~mm} / \mathrm{a}$. In the east of monitor, the area of Chaoyang and Tongzhou, there are obvious subsidence phenomenon。This subsidence is affected by groundwater exploitation, flake subsidence it had an tendency of expanding to the east and south, and the maximum annual subsidence is more than $76 \mathrm{~mm} /$ year. The results of this monitoring are basically consistent with the published data of surface subsidence in Beijing.
\end{abstract}

\section{INTRODUCTION}

Due to a great deal of groundwater exploitation, serious surface subsidence occurred in some areas of Beijing. Surface subsidence is a long and slow process with reaction hysteresis, which is difficult to be detected and monitored in a short time range, but once it happens, the damage is unbearable to contemplate. For the city, surface subsidence causes the damage of urban piping system and drainage system, such as pipe distortion and deformation fracture, which seriously affects the safety of production and normal life.
Interferometric Point Target Analysis (IPTA) can achieve high precision inversion of surface deformation by processing the phase information on the stable point target and maximum overcoming the influence of decorrelation factors and atmospheric delay. It has the advantages of short monitoring period, wide coverage and low application cost.

\section{IPTA TECHNOLOGY}

IPTA technology is an improvement of PS-InSAR technology in the direction of data storage and data processing. It uses the 
permanent scatterers (PS) points identified on the time series SAR interferogram to establish a permanent scatterer network with time series stable and highly reflective features(Yu $\mathrm{X}$, 2012a). It calculates and separates the baseline error phase, the DEM residual phase, the atmospheric phase, the surface offset phase and so on by the iterative processing of the unwrapping phase of the difference interferogram. Thus, the high-precision surface deformation variables and the accurate DEM model of the region are obtained. There are two main advantages of IPTA technology, one is the refinement of vertical baseline information and the iterative solution of deformation parameter and elevation correction, another is that all data processing processes are completed in vector mode, greatly reducing the amount of data and improving the efficiency of data processing(Zhang H, 2016). In IPTA processing, the interference phase can be decomposed into:

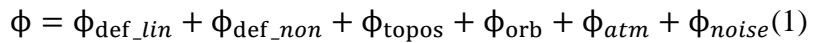

where $\emptyset=$ Point target interference phase

$\phi_{\text {def_lin }_{\text {L }}}, \phi_{\text {def }_{-} n o n}=$ The linear and nonlinear deformation phases on the radar line of sight (LOS)

$\phi_{\text {topos }}=$ Inaccurate DEM caused by the residual terrain phase

$\phi_{\text {orb }}=$ The residual phase caused by the track error

$\phi_{a t m}=$ Atmospheric delay phase

$\phi_{\text {noise }}=$ System thermal noise

IPTA technology is a process of modifying the model parameters from coarse to fine by constantly iterating. It uses initial parameter estimation model to generate model phase, and analyzes residual error to extract elevation error and shape change rate correction. The model parameters are improved by the correction value of the solution, and the nonlinear deformation and the separation of the atmospheric phase are carried out according to the different spatial and temporal characteristics of each phase component. The improved model parameters are further improved by this idea, so that the phase of each part is closer to the true value. The specific process shown in Figure 1(Zhang H, 2016).

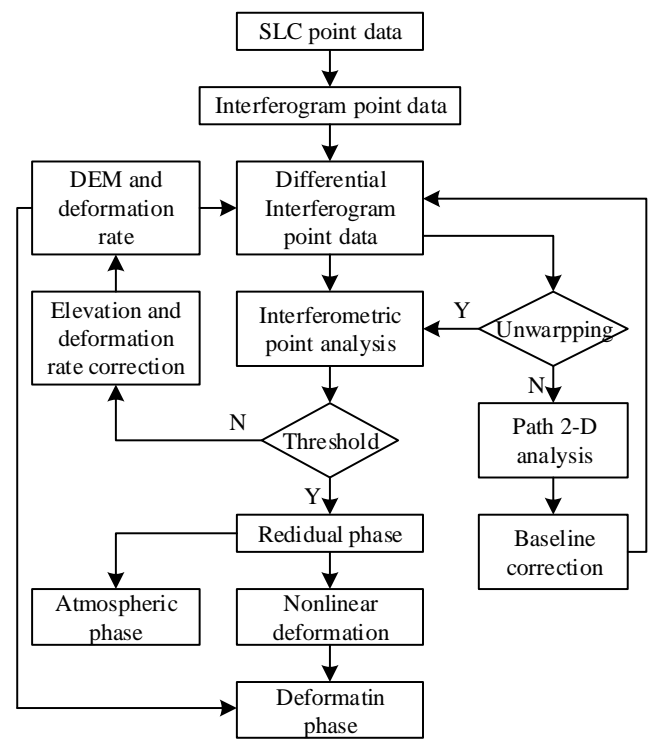

Figure 1 IPTA flow chart

\section{IPTA TECHNOLOGY KEY STEPS}

\subsection{PS Point Selection}

The PS point target is considered as a good coherent target, and it can maintain a good frontal stability for a long time and can reflect the true deformation of the ground surface. The PS point detection methods used in IPTA are amplitude dispersion index thresholding method and correlation coefficient thresholding method.

On the high signal-noise ratio pixel, the phase noise level can be measured using the temporal amplitude information. The phase standard deviation of the PS points based on the high signal-tonoise ratio (SNR) approximates the amplitude deviation index, therefore the PS point is selected by the reciprocal of amplitude dispersion index thresholding (MSR)( Yu X, 2012b and Ding W, 2011).

$$
\operatorname{MSR}=\frac{m_{A}}{\sigma_{A}}
$$

where $m_{A}=$ Time series amplitude mean value

$\sigma_{A}=$ Time series standard deviation

Another method, IPTA takes the mean value of the coherent graph of the time series and selects the PS points by using the time series averaged coherence value $\left(\gamma_{\text {mean }}\right)(\mathrm{Yu} X, 2012 \mathrm{~b})$. 


$$
\gamma_{\text {mean }}=\frac{1}{M} \sum_{j=1}^{M} \gamma_{j}
$$

where $\gamma_{\text {mean }}=$ The coherent graph of the jth interferogram $\mathrm{M}=$ Number of interferograms

\subsection{Elevation Eorrection and Linear Deformation Rate Solution}

The second difference of the differential phase of the adjacent point target can weaken the influence of the atmospheric delay phase and the nonlinear deformation phase. The quadratic differential phase model based on the adjacent point target can be obtained by combining the equation (1) (Zhang H, 2016).

$$
\Delta \phi_{i, j}=\frac{-4 \pi B_{\perp}}{\lambda R \sin \theta} \Delta \varepsilon_{i, j}-\frac{4 \pi}{\lambda} \Delta \mathrm{v}_{i, j} t+\Delta \phi_{r e s, i, j}
$$

where $\Delta \varepsilon_{i, j}=$ Elevation correction of adjacent point target

$\Delta \mathrm{v}_{i, j}=$ Deformation rate difference of adjacent point target $\Delta \phi_{\text {res }, i, j}=$ The residual phase difference including nonlinear deformation, atmospheric phase and noise phase

In order to solve $\Delta \varepsilon_{i, j}$ and $\Delta \mathrm{v}_{i, j}$, the whole time coherence coefficient is introduced.

$$
\gamma_{i, j}=\left|\frac{1}{M} \sum_{m=1}^{M} \exp \left(j \cdot \Delta \phi_{r e s, i, j}\right)\right|
$$

The differential interferogram is analyzed by temporal two dimensional regression analysis. The $\Delta \varepsilon_{i, j}$ and $\Delta \mathrm{v}_{i, j}$ values of $\gamma_{i, j}$ are obtained as the estimated parameters. Then we construct an irregular triangulation with a limited side length and select the more stable point in the study area as the reference point. We use the regional network adjustment method to calculate the elevation error correction value and the shape change rate correction value of each vertex in the triangulation network relative to the reference point.

\subsection{Baseline Refinement}

Orbital error is the baseline error in InSAR. The baseline error phase is linearly related to the spatial scale and exists in the form of residual fringes and in the interferogram. Therefore, it is important to refine the baseline to improve the accuracy of deformation parameters. IPTA uses the least square method to refine the baseline vectors based on the corrected elevation values and the unwrapping phase information. In this paper, first of all, we remove the phase noise of the residual phase by using the spatial filtering. Then the Minmum Cost Flow (MCF) method is used to unwarp the residual phase and obtain the more accurate unwrapping phase. Finally, the error equations are established for the unwrapping phase based on the corrected elevation values and the baseline parameters are estimated by the least squares method.

\subsection{Atmospheric Phase and Nonlinear Deformation Phase Separation}

The residual phase mainly includes nonlinear deformation, atmospheric phase and noise. In general, the atmospheric delay phase can be treated as a temporally high-frequency signal and the nonlinear deformation phase as a low-frequency signal that accumulates during a timespan comparable to that of the interferogram (Ferretti A, 2001 and Berardino P, 2002.)。 However the atmospheric delay phase have relatively low spatial frequency. This is the basis for multi-temporal InSAR processing, which can be used to separate the effects of tropospheric delays and nonlinear ground surface deformation。

\section{DATA PROCESSING ANALYSIS}

\subsection{Experimental Data and Area}

In this paper, 20 images of the TerraSAR-X data and the highprecision DSM product WorldDEM produced by DLR in Beijing were processed by using IPTA technology, and the results of surface subsidence from October 2016 to June 2017 were obtained and analyzed.

The imaging mode of TerraSAR-X data used in this experiment is StripMap with a resolution of 3 meters and a revisiting period of 11 days. In addition, the resolution of the high-precision WorldDEM DSM products is 12 meters. The elevation accuracy is better than $2 \mathrm{~m}$ in flat area and is better than $4 \mathrm{~m}$ in the mountain. The horizontal precision is better than $10 \mathrm{~m}$. Using worldDEM can improve the precision of analog phase, reduce the influence of elevation error and obtain more reliable inversion results. Due 
to the impact of SAR and the influence of DSM coverage, the SAR images were cut and selected in the area of about $700 \mathrm{~km}^{2}$ in Beijing. The coverage area of the SAR image is shown in Figure 2.

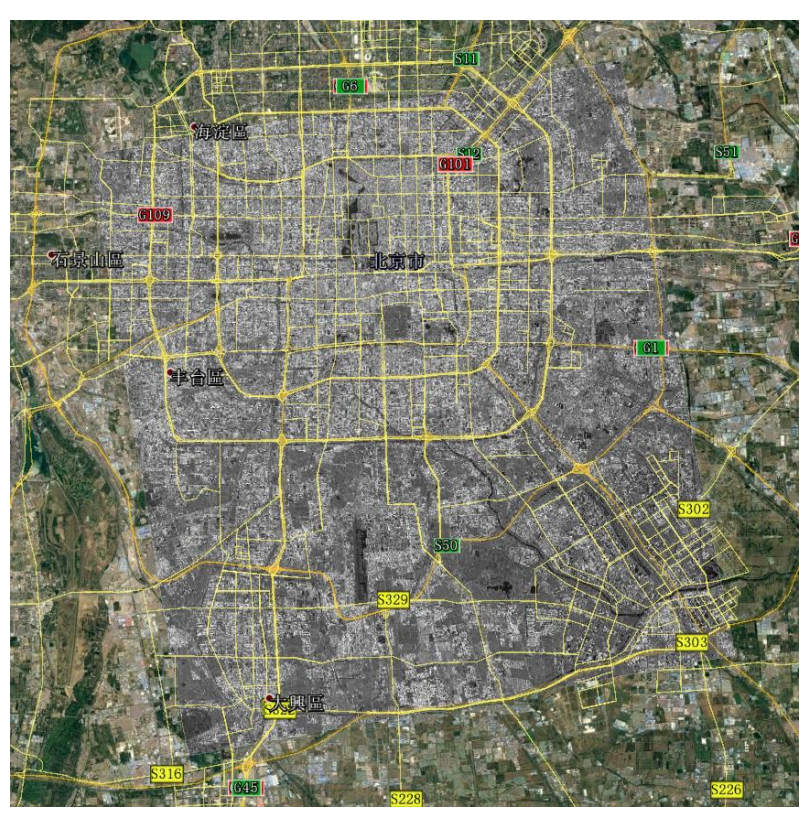

Figure 2.Coverage area of SAR image

In order to ensure high temporal coherence of interferogram, in the acquired 20 scene of TerraSAR-X data, we select 3 scene images of adjacent time as a set of a small baseline set which consists of 70 pairs of interference pairs. The interference connection diagram is shown in Fig 3. X represents the time base of each interference pair, and Y represents the vertical baseline of each interference pair.

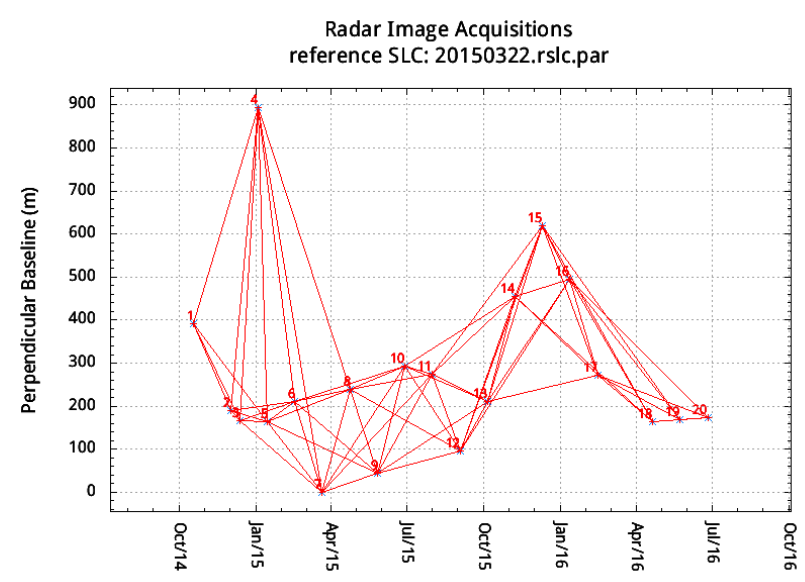

Figure 3. Connection diagram

\subsection{PS Point Selection}

In this experiment, the PS point is selected by Applying the method of Section 2.3, and the threshold of the selected point is $($ MSR $>2) \cap\left(\gamma_{\text {mean }}>0.5\right)$, and 1462710 initial candidates are selected, as shown in Figure 4. As can be seen from the diagram, the PS points screened under the higher MSR and $\gamma_{\text {mean }}$ thresholds are mainly distributed on artificial buildings such as roads and houses. There is almost no point in rivers, lakes and other waters. It shows that there are abundant and high quality targets in urban area. Due to the short wavelength of the $\mathrm{X}$-band of SAR image, the interference pattern has low coherence in natural area such as farmland and woodland, so there are few PS points.

\subsection{Result and Analysis}

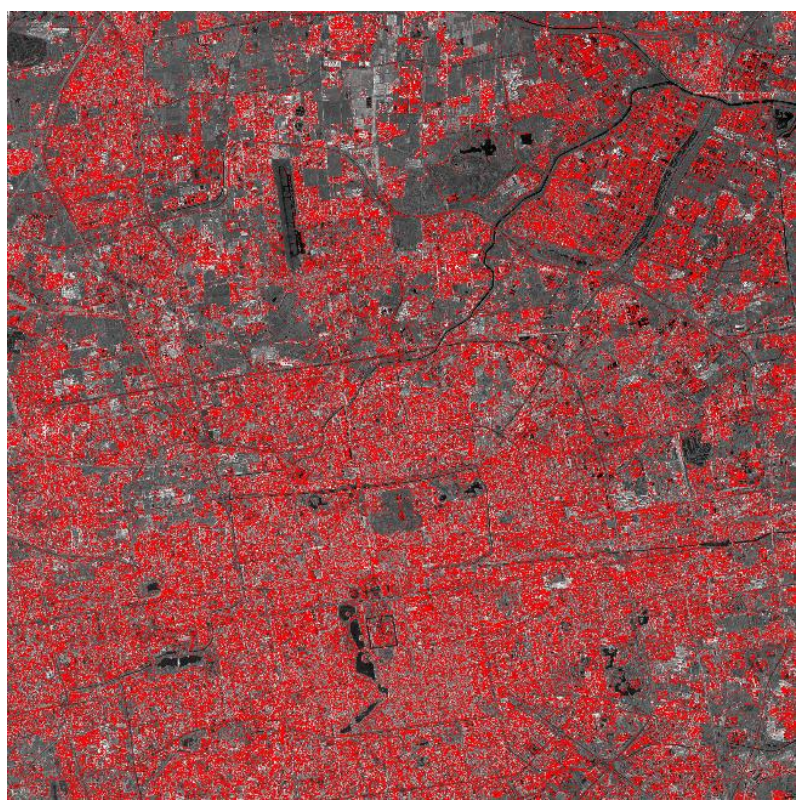

Figure 4. Distribution map of the initial candidate point

The results of surface subsidence monitoring in Beijing area using IPTA technique are shown in figure 5. As can be seen from the diagram, most of the ground surface (Beijing City Center) in the monitoring area is relatively stable in monitoring time, and there is a large area of surface (Eastern Beijing city) subsidence on the left side of the monitoring area. From the north to the south a large sheet deformation zone (Chaoyang Yuegezhuang Tongzhou Anding camp) has formed. The deformation area is about $25 \mathrm{~km}$ in the north-south direction and the widest part is 
$7.5 \mathrm{~km}$ in the east-west direction. The total area is about $90 \mathrm{~km}^{2}$

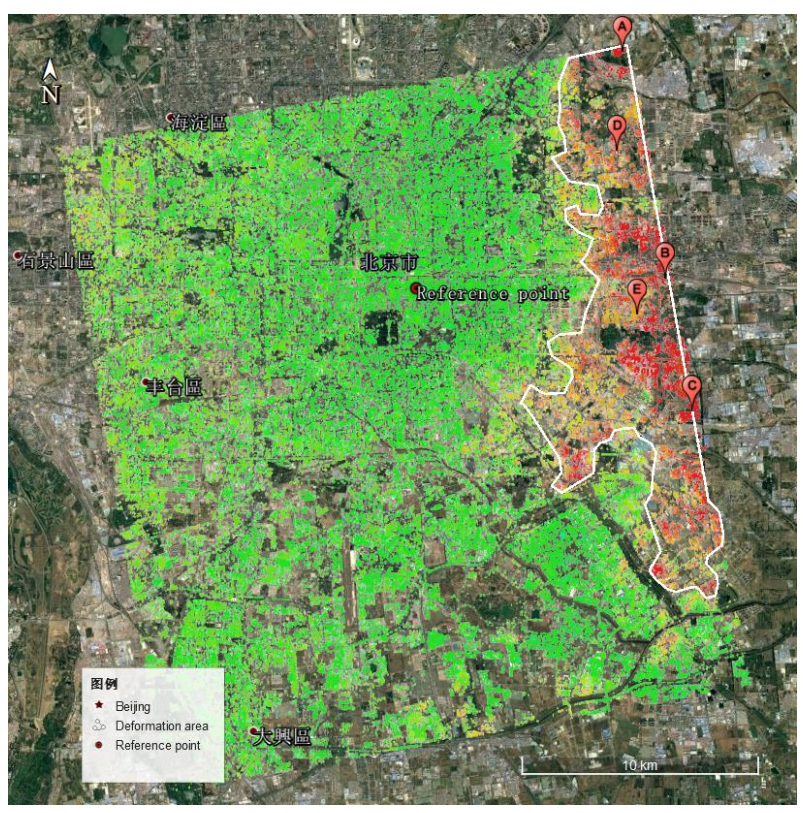

Figure 5. Surface deformation results monitoring area are interpolated, and the contour map of the accumulated deformation variables in the monitoring area is drawn, as shown in figure 6 . As can be seen from the diagram, there are three deformation centers in the monitoring area, corresponding to the three points of $\mathrm{A}, \mathrm{B}$ and $\mathrm{C}$ in figure 3 . Point $\mathrm{D}$ and $\mathrm{E}$ are the deformation points in the center of the two deformation centers. Point $\mathrm{A}, \mathrm{B}, \mathrm{C}, \mathrm{D}$ and $\mathrm{E}$ relative to the reference point cumulative settlement are $-129 \mathrm{~m},-120 \mathrm{~mm}$,$126 \mathrm{~mm},-60 \mathrm{~mm}$ and $-54 \mathrm{~mm}$. The settling rates of $\mathrm{A}, \mathrm{B}$, and $\mathrm{C}$ relative to the reference point are $-76 \mathrm{~mm} /$ year, $-70 \mathrm{~mm} /$ year, $75 \mathrm{~mm} /$ year, $-33 \mathrm{~mm} /$ year and $-30 \mathrm{~mm} /$ year. Therefore, the maximum cumulative shape variable in this region exceeds $129 \mathrm{~mm}$ and the maximum strain rate exceeds $76 \mathrm{~mm} /$ year. Data show that nearly half of Beijing's water supply comes from underground water. The groundwater level was dropped by one meter from 2011 to 2015 . The deformation area is located in the CBD area of Beijing. With a large population, huge water consumption and unique geological conditions, the region is seriously subsiding(Chen B, 2017).

The accumulated deformation values of all PS points in the

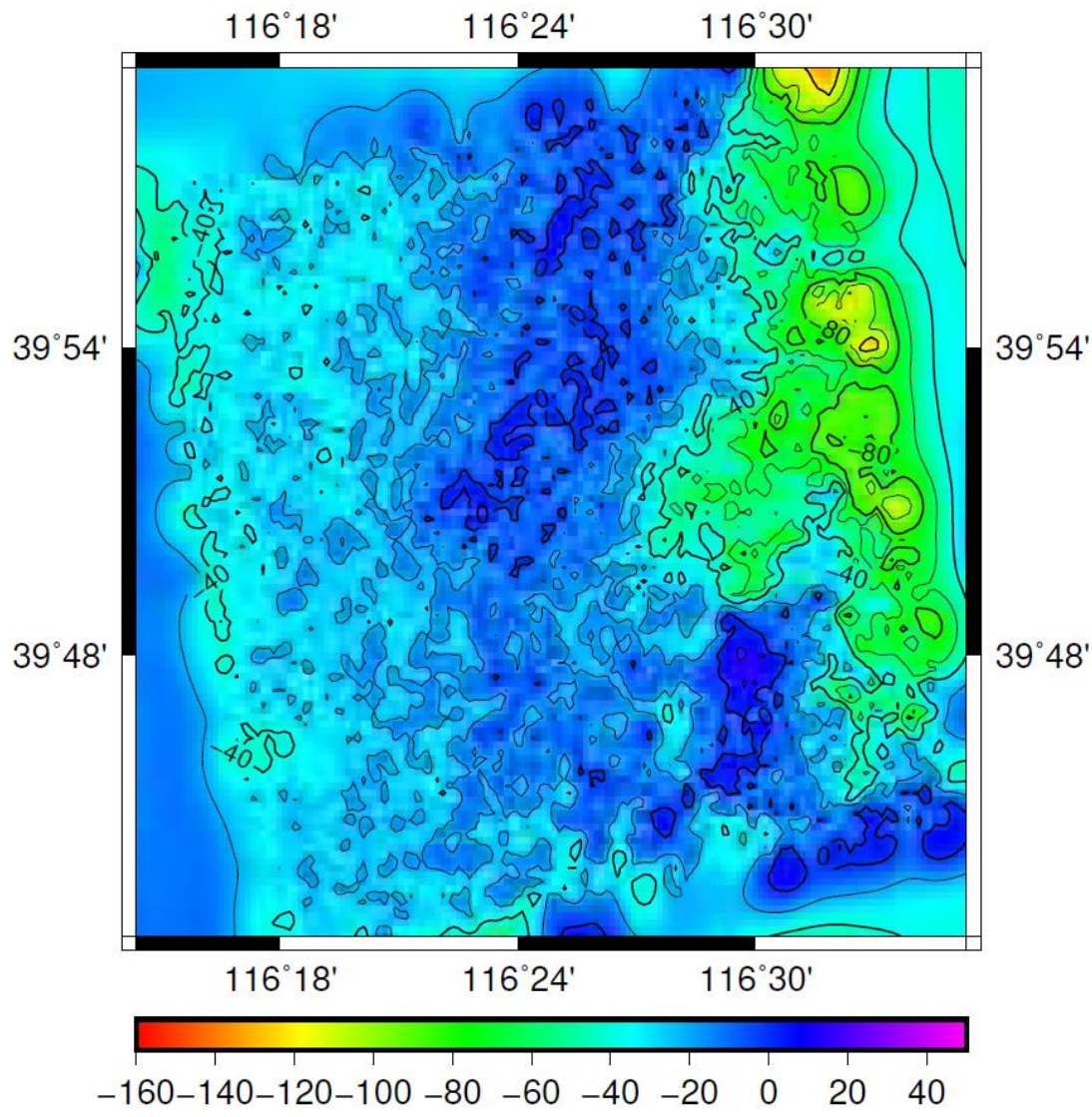

Figure 6. Contour map 


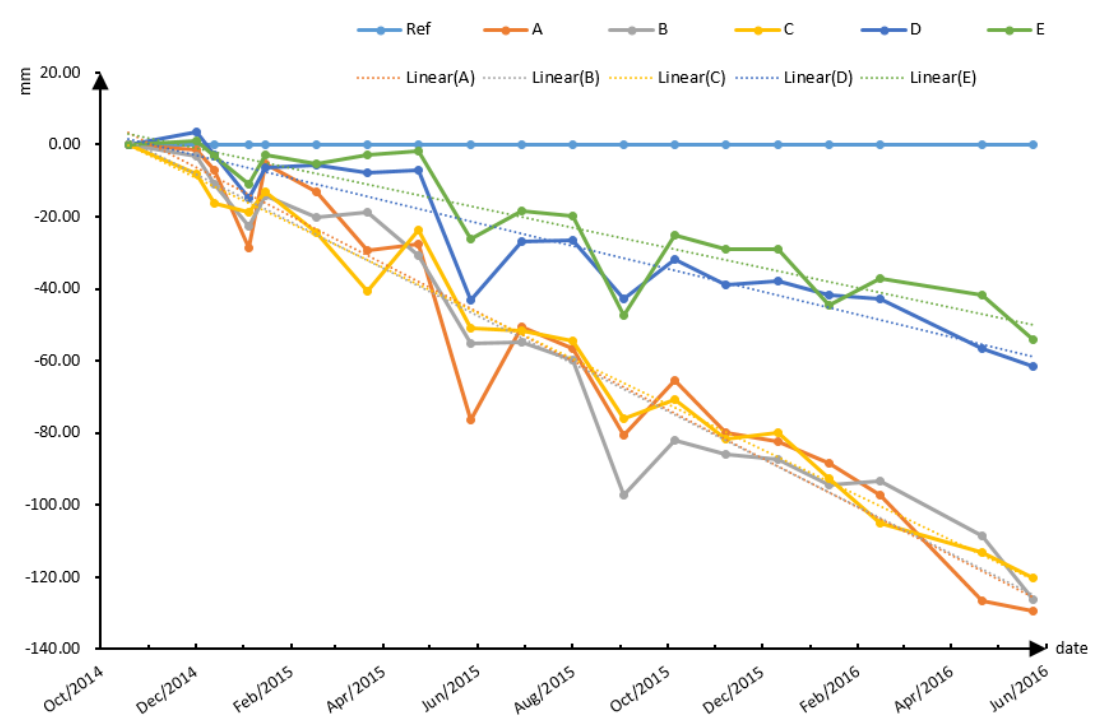

Figure 7. Cumulative deformation of the line graph

Figure 7 is A, B, C, D, E five points relative to the reference point of the cumulative deformation of the line graph. As can be seen from the diagram, the deformation tendencies of $\mathrm{A}, \mathrm{B}$ and $\mathrm{C}$ are consistent, and the deformation tendencies of $\mathrm{D}$ and $\mathrm{E}$ are consistent. However, the deformations of $\mathrm{D}$ and $\mathrm{E}$ have a lag in time relative to the deformation center. D and $\mathrm{E}$ were basically stable before July 2015 and no obvious deformation occurred. After July 2015, obvious deformation occurred. The above phenomena indicate that the deformations of the region first occur at A, B, C. As time goes by, the deformation range is expanding, so that three independent deformation points are connected to form a large area of sheet deformation area. And the range of deformation still has the trend of enlargement.

\section{CONCLUSION}

In this paper, we used IPTA technology to deal with 20 scenes of TerraSAX-X SAR images of Beijing and monitored three deformation centers. The maximum cumulative shape variable exceeds $129 \mathrm{~mm}$ and the maximum strain rate exceeds $76 \mathrm{~mm} /$ year And the range of deformation still has the trend of enlargement. The impact of groundwater exploitation on urban surface subsidence is still serious. At the same time, due to the limited data, this paper does not verify the accuracy of the monitoring results, and later I will collect other monitoring data for verification. In addition, there are some error phases in data processing which cannot be effectively separated and eliminated. More accurate solution model is the focus of subsequent research.

\section{REFERENCES}

Berardino P, J., 2002. A new algorithm for surface deformation monitoring based on small baseline differential SAR interferograms. IEEE Transactions on Geoscience \& Remote Sensing, 2003, 40(11):2375-2383.

Chen B, J., 2017 Characterization and causes of land subsidence in Beijing, China. International Journal of Remote Sensing, 38(3), pp.808-826.

Ding W, D., 2011. Study of PSInSAR on the technique of points selection and phase unwrapping. Central South University.

Ferretti A, J., 2001. Permanent scatterers in SAR interferometry. IEEE Transactions on Geoscience \& Remote Sensing, 39(1), p.820.

Yu X, J., 2012a. Application of IPTA method in surface subsidence monitoring of San Pedro Bay Port. Science and Surveying and Mapping, 37(6), pp.21-25

Yu X, D., 2012b. Improved SBAS technology for land deformation detection and groundwater application. Central South University.

Zhang H, J., 2016. Application of IPTA method in surface subsidence monitoring. Journal of Geomatics Science and Technology, 33(2), pp.145-149. 